\title{
Gene network analysis of complex diseases using GenCoNet
}

\author{
O. Zolotareva ${ }^{1,2}$, A. Shoshi ${ }^{1}$, R. Hofestädt ${ }^{1}$, A. Maier ${ }^{1}$, V. Ivanisenko ${ }^{3}$,V. Dosenko ${ }^{4}$, \\ E. Bragina ${ }^{5}$ \\ ${ }^{1}$ Bielefeld University, Bioinformatics / Medical Informatics Department, Bielefeld, Germany \\ ${ }^{2}$ Bielefeld University, International Research Group "Computational Methods for the Analysis \\ of the Diversity and Dynamics of Genoms", Bielefeld, Germany \\ ${ }^{3}$ Institute of Cytology and Genetics SB RAS, Novosibirsk, Russia \\ ${ }^{4}$ Bogomoletz Institute of Physiology, Kiev, Ukraine \\ ${ }^{5}$ Research Institute of Medical Genetics, Tomsk NRMC, Tomsk, Russia \\ * e-mail: ralf.hofestaedt@uni-bielefeld.de
}

Key words: network analysis, database system, disease

Motivation and Aim: Complex diseases affect millions of people worldwide and impose individuals and society a huge socioeconomic burden. Although complex diseases cannot be explained by the Mendelian model of inheritance, certain molecular-genetic components in their pathogenesis are expected. Moreover, since many of complex diseases are comorbid, i.e. demonstrate higher than expected co-occurrence, may be assumed to share molecular players and mechanisms. Identification of these shared molecular players and mechanisms in the pathogenesis of comorbid diseases is necessary for selection of the most appropriate treatment strategy. To address this ongoing need, the Neo4j database "GeCoNet" was developed which integrates various associations between diseases, genes, variants and chemical compounds for four diseases that have a strong molecular-genetic component and demonstrate comorbidity: asthma, essential hypertension, diabetes and Alzheimer's disease.

GenCoNet is meant to be a qualitative resource that facilitates researchers access the relevant information. Our study [1] showed that the application of methods of reconstruction and analysis of gene networks is a productive tool to rank candidate genes by their importance to complex diseases (e.g. asthma and hypertension).

Acknowledgements: Supported by the Volkswagenstiftung grant No. 90335.

\section{References}

1. Saik O.V., Demenkov P.S., Ivanisenko T.V., Bragina E.Y., Freidin M.B., Gonchareva I.A., Dosenko V.E., Zolotareva O., Hofestädt R., Lavrik I.N., Rogaev E.1., Ivanisenko V.A. (2018) Novel candidate genes important for asthma and hypertension comorbidity revealed from associative gene networks. BMC Med Genomics. 11(1):15. 